\title{
Obstetric Hemorrhage and Hypothermia: Chilling Facts
}

\author{
Wayne R. Cohen \\ Department of Obstetrics and Gynecology, University of Arizona College of Medicine, Tucson, AZ, USA \\ Email: waynercohen@me.com
}

How to cite this paper: Cohen, W.R. (2018) Obstetric Hemorrhage and Hypothermia: Chilling Facts. Open Journal of Obstetrics and Gynecology, 8, 1260-1270. https://doi.org/10.4236/ojog.2018.813128

Received: August 26, 2018

Accepted: October 27, 2018

Published: October 30, 2018

Copyright (C) 2018 by author and Scientific Research Publishing Inc. This work is licensed under the Creative Commons Attribution International License (CC BY 4.0).

http://creativecommons.org/licenses/by/4.0/

\section{(c) (i) Open Access}

\begin{abstract}
Objective: This clinical perspective reviews the causes, prevention and treatment of accidental hypothermia in severe obstetric hemorrhage. Results: Hypothermia commonly accompanies hemorrhagic shock. Hypothermia can inhibit blood coagulation, reduce cardiac contractility, predispose to arrhythmias, contribute to acidosis, and suppress immune function. Several techniques for warming a patient or reducing heat loss are available. Keeping the patient dry, covering her with blankets, and raising the ambient temperature in the room are valuable. Methods to transmit heat actively are more effective. Forced warm air blowers are efficient. Heating intravenous fluids is important, and warm fluid lavage of the open abdomen can be effective. Conclusion: Monitoring core temperature in the operating room and choosing therapy is a shared responsibility of surgeon and anesthesiologist.
\end{abstract}

\section{Keywords}

Coagulopathy, Hemorrhage, Hemorrhagic Shock, Hypothermia, Postpartum Hemorrhage

\section{Introduction}

New insights into the pathophysiology of hypovolemic shock, and the incorporation of that knowledge into innovative paradigms for management, have led to improved outcomes for patients with exsanguinating hemorrhage. Prompt recognition of hypovolemia, appropriate fluid resuscitation with early and generous infusion of blood components, application of proper surgical techniques, and the introduction of new therapies have all contributed to better results.

Despite this progress, severe hemorrhage complicating pregnancy and the postpartum period remains a major cause of maternal death and severe morbidity. One contributor to this devastation is the loss of thermal homeostasis in the 
bleeding patient. Hypothermia is, in fact, an insidious and common accompaniment of severe hemorrhage, but is often overlooked as a factor in the failure to recover from serious obstetric bleeding.

Recognition of the adverse consequences of hypothermia has been an important component in modifying our approach to the actively bleeding patient, and the maintenance of euthermia should be considered a primary goal in the management of women with severe obstetric hemorrhage. Indeed, even if all other aspects of care have been well managed, unresolved hypothermia can render the outcome solemn.

Relatively scant attention has been devoted to characterizing and analyzing massive obstetric hemorrhage. Its pathophysiology may differ in some respects from hemorrhage associated with major trauma, which usually occurs remote from medical care and includes extensive tissue injury. The basic cellular mechanisms underlying uterine bleeding are not well understood, and optimal directed therapy awaits such insights. Nevertheless, all forms of severe bleeding share certain features, and among these, loss of body heat is of critical importance.

\section{Measurement of Body Temperature}

The best reflection of overall thermal homeostasis is core temperature. Optimal sites for its measurement are the pulmonary artery, distal esophagus, tympanic membrane and nasopharynx [1]. Other options, although less accurate but clinically useful when optimal sites are unavailable, are the rectum, mouth, axilla, and skin surface.

Mercury- or alcohol-in-glass thermometers should not be used in acute situations. They are slow and inconvenient, and break easily. Infrared techniques have become commonplace, particularly in ear thermometers, and, if directed at the tympanic membrane and not other areas of the ear canal, determine core temperature accurately. Many of today's electronic thermometers utilize disposable thermistors or thermocouples. A relatively new technique, deep temperature monitoring, provides accurate estimates of core temperature from the skin and is quite convenient [2].

\section{Causes of Hypothermia}

The obstetric patient with hemorrhage is especially vulnerable to heat loss. During efforts to manage her problem she is usually immobile, obviating heat production by muscular activity. Often large areas of skin are exposed, and her gown and bedclothes may be wet, leading to evaporative and radiant heat dissipation. She may be anesthetized, and her abdomen open, further abetting thermal loss. Moreover, with good intent, bleeding patients often receive rapidly infused large volumes of room temperature crystalloids, or blood components recently removed from refrigeration. About six liters of room temperature fluid administered intravenously will drop core body temperature by as much as $2^{\circ} \mathrm{C}$, 
as will several units of cooled packed red blood cells infused rapidly. Temperature may fall much more quickly if thermoregulation is impaired by anesthesia or other drugs.

The aggregation of these factors tends to drive body temperature down with surprising rapidity. Hypothermia, even when relatively mild, can have serious, even fatal, consequences for the patient with hemorrhage by potentiating many of the serious elements of hypovolemic shock (Table 1) [3] [4]. Some of these complications occur primarily with severe hypothermia or in patients with predisposing illnesses or metabolic aberrations. Many can, however, develop in the context of relatively mild falls in core temperature.

\section{Pathophysiology}

Thermoregulation during pregnancy, particularly as it involves accidental hypothermia and severe hemorrhage, has not been well studied. There is reason to believe that pregnancy might alter thermoregulation because sympathoadrenal responses to heat loss and recovery from shock are influenced by sex hormones [5] [6]. Moreover, baseline volume and coagulation status are altered. Nevertheless, most of the principles we apply to pregnant (or recently pregnant) women are drawn from clinical experience and from studies done on nonpregnant individuals or experimental animals.

Most physiologic processes are programmed to work efficiently within a narrow temperature spectrum. Under normal circumstances, a complex and sensitive set of thermoregulatory mechanisms maintains core temperature within a limited range (usually $37^{\circ} \mathrm{C} \pm 1^{\circ} \mathrm{C}$ ) by precisely regulating heat loss and generation. The normal core temperature in humans is generally considered $36.5^{\circ} \mathrm{C}$ $37.5^{\circ} \mathrm{C}$.

Dangerous falls in core temperature are usually prevented by several defenses, mediated predominantly through the hypothalamus: [7] behavioral change (increasing physical activity, moving to a warmer environment, etc.); adrenergically

Table 1. Complications associated with accidental hypothermia.

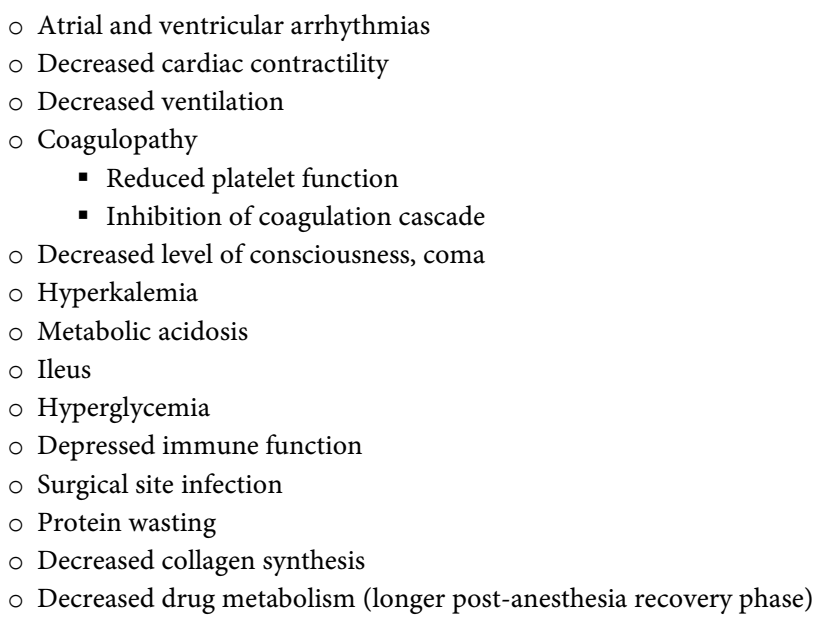


modulated peripheral vasoconstriction; and shivering. All are geared to increase metabolic heat generation. (Nonshivering thermogenesis in brown fat is of minimal importance in adults.) Each of these regulatory defenses can be inhibited during the management of hemorrhage, when, as noted, several things occur that promote rapid heat loss. A core temperature $<36^{\circ} \mathrm{C}$ indicates that thermal stress has overcome physiologic defenses, and is clinically considered hypothermia.

Normal systemic blood flow, often compromised during severe hemorrhage, is an important component of thermal homeostasis. Heat is delivered to peripheral tissues from the central circulation, and deficient blood flow during shock can potentiate peripheral cooling. Heat loss from the skin surface is mediated primarily through arteriovenous shunts in acral cutaneous vasculature. The diameter of these shunts is controlled by alpha sympathetic stimulation in response to changes in central temperature in the hypothalamus. Some anesthetics and other drugs (and hypothermia itself) that inhibit sympathetic nervous system responses will thereby perpetuate heat loss.

Hypothermia can be problematic in the bleeding patient for a number of reasons (Table 2). Of greatest concern is the inhibition blood coagulation. Also, reduced temperature shifts the position of the oxyhemoglobin dissociation curve leftward, increasing the affinity of hemoglobin for oxygen. Therefore, oxygen is released less readily to tissues. Cardiac contractility is diminished by hypothermia, and the risk of ventricular arrhythmias is increased. Consciousness may be depressed and acidosis promoted. With severe hypothermia adrenergic agonists like epinephrine, norepinephrine and isoproterenol, often used in the management of shock, do not support cardiac function as they do at normal core temperatures [8].

\subsection{Coagulopathy}

Coagulation defects associated with hypovolemic shock and hypothermia often resist all attempts at reversal as long as body temperature remains low. Although the adverse effects of accidental hypothermia during hemorrhage are most marked

Table 2. Methods for prevention and treatment of hypothermia.

- Prevention of Heat Loss

- Blankets (fabric or thermal)

- Increase ambient temperature

- Keep patient dry

- Provision of Additional Heat

o Forced warm air heater

- Circulating warm water garment

- Body cavity lavage

- Hollow organ lavage (stomach, bladder)

- Warmed intravenous fluids

- Heated inspired air

- Cardiopulmonary bypass

- Extracorporeal membrane oxygenation

o Continuous veno-venous rewarming 
when the temperature fall is severe, even mild hypothermia of less than $1^{\circ} \mathrm{C}$ increases surgical blood loss significantly [3].

Coagulopathy during shock is usually contributed to by several mechanisms [9] [10] [11]. Dilution of circulating clotting factors occurs when large volumes of factor-deficient fluids are administered to replace lost blood. Hypoperfusion, acidosis and hypothermia each contribute to the coagulopathy, and consumption of clotting factors can occur in rapidly growing hematomas such as may be found in the uterine cavity or elsewhere in the pelvis. The shock state results in hypoperfusion, which increases endothelial thrombomodulin expression, thereby activating anticoagulant proteins, and contributing to hemorrhage [11]. Also, sludging or clotting may occur in markedly vasoconstricted, underperfused vascular beds. Disseminated intravascular coagulation (DIC) may supervene.

Hypothermia can cause or abet clotting aberrations in other important ways. It decreases temperature-dependent enzyme activity in the coagulation cascade, [10] [12], inhibits platelet activity [12], and reduces fibrinogen and thrombin availability [13]. It is vital to recognize that substantial temperature-related degradation of coagulation may not be recognized in tests such as the prothrombin time, the partial thromboplastin time, or thromboelastography, which are routinely performed in the laboratory at $37^{\circ} \mathrm{C}$. Attention to clinical signs of a coagulopathy, including evidence of anemia, hemolysis and development of new bleeding sites is therefore of paramount importance. When coagulation factor concentrations are diminished by dilution or consumption, or if acidemia is present, the effect of hypothermia may be magnified.

In addition to increasing fibrinolysis, and decreasing production of coagulation factors, hypothermia inhibits platelet production of thromboxane A2, which promotes platelet aggregation and vasoconstriction. Even coagulation processes that are not enzyme-dependent are affected by hypothermia. For example, the interaction of platelets with von Willebrand factor is inhibited.

\subsection{The Lethal Triad}

The term "lethal triad" refers to a situation in which coagulopathy, acidosis and hypothermia occur in association with major traumatic blood loss. This combination confers an exceptionally high mortality. It can occur in obstetric hemorrhage as well and demands prevention or prompt correction if the mother is to be saved. The three conditions appear to be synergistic, and feed upon one another, leading rapidly to an irreversible shock state.

\subsection{Other Morbidities}

Data demonstrate convincingly that falls in temperature of only about $2^{\circ} \mathrm{C}$ can be associated with myocardial injury and other cardiovascular morbidity [14]. Perioperative hypothermia may also have consequences for post-surgical complications. It can delay metabolism and excretion of drugs and toxins and delay recovery from anesthetics [15]. Moreover, it may impair wound healing and promote the development of infectious complications through immunosuppression [16]. 


\section{Role of Anesthesia}

General and neuraxial anesthesia contribute to the development of hypothermia by compromising normal thermoregulatory responses [4] [17]. Peripheral vasodilatation increases heat loss, and anesthetized patients cannot generate heat by increasing muscular activity. Under general anesthesia vasoconstriction and shivering may be restrained, and patients become essential poikilotherms, with core temperature heavily dependent on ambient conditions. Regional anesthesia is similarly problematic, although its effects are generally more moderate than those of general anesthesia. Neuraxial blocks cause vasodilatation and shivering through both peripheral and central mechanisms.

Other drugs commonly used in conjunction with anesthetics may compromise thermal regulation by inhibiting movement or shivering (muscle relaxants) or vasoconstriction (vasodilators), and sedatives or analgesics may have synergistic effects with neuraxial agents, at least in the elderly. Hypothermia may prolong recovery from anesthesia by reducing the rate of drug metabolism.

\section{Prevention and Treatment}

Like most problems of disturbed homeostasis, loss of body heat is easier to prevent than to treat, and routine patient warming is warranted, effective, and safe during major surgical procedures, including cesarean delivery [18] [19]. Whenever a patient is in a situation in which substantial fluid resuscitation is likely to be necessary, prophylactic efforts to prevent heat loss should be employed (Figure 1).

Several techniques for warming a patient or reducing heat loss are available (Table 2). Replacement of heat can take considerable time intraoperatively or postoperatively, during which the patient remains vulnerable to the adverse effects of reduced temperature. Prompt efforts to produce euthermia are therefore critically important. Generally, several approaches need to be used simultaneously to achieve efficient restoration of core temperature.

Dry blankets are valuable. (Although warm blankets are often used, it is uncertain whether they provide significant benefits over those kept at ambient temperature.) Special insulating blankets are available. Raising the temperature in the operating or delivery room to decrease the gradient between patient and air is somewhat helpful, but can make the care team quite uncomfortable. These forms of heat loss prevention, which allow the body's intrinsic heat generation to correct deficient core temperature, should only be used alone when hypothermia is mild, blood loss is controlled, and no factors such as anesthesia are present to inhibit normal thermal homeostasis. Active methods to transfer heat to the patient are more likely to be effective.

Forced warm air systems are in widespread use and are an effective means for transferring heat to the body [20]. They can be used prophylactically or therapeutically. They are more efficient than blankets, even those that incorporate warm water. Warmed inspired humidified air can be a source of heat as well. 


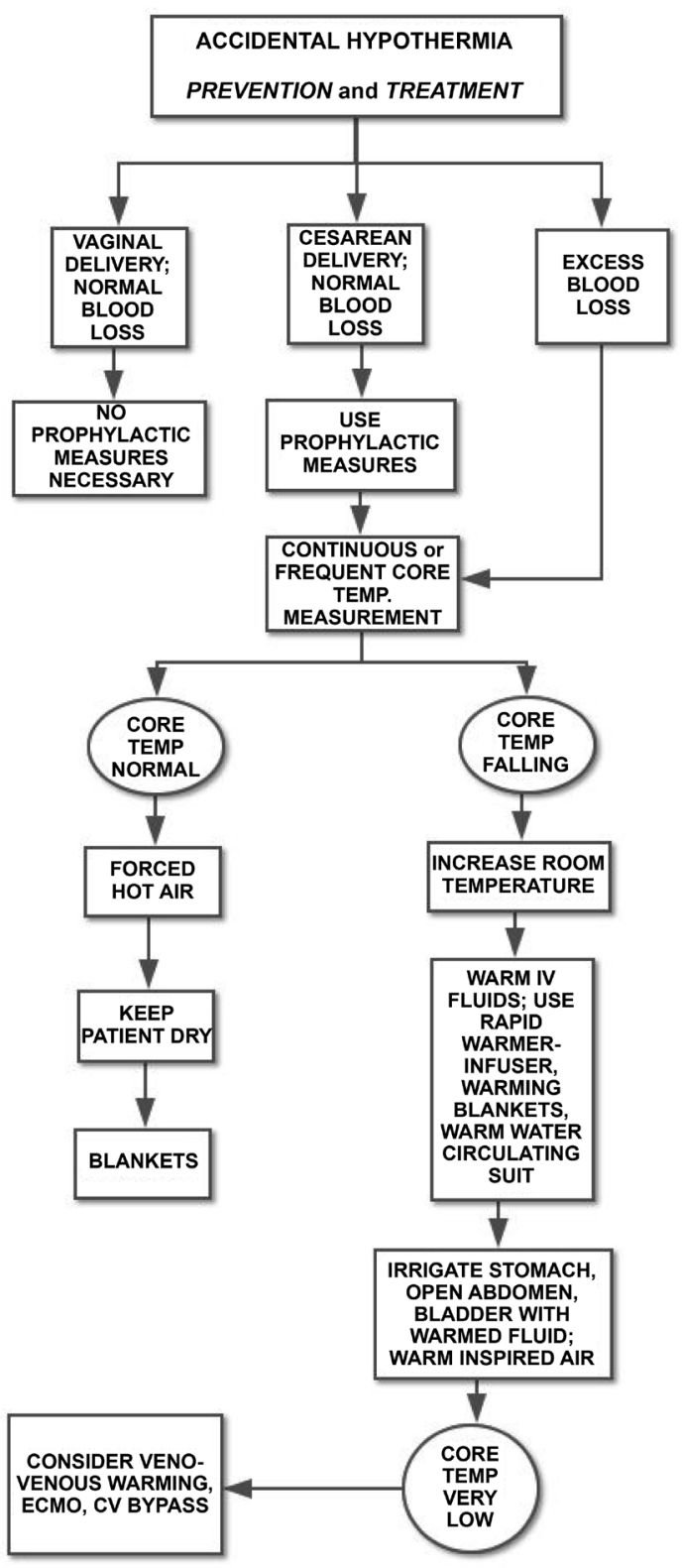

Figure 1. Prophylactic measures to prevent heat loss should be used during all cesarean deliveries. When blood loss is excessive after any delivery or core temperature is falling, providing extra heat energy to the patient is necessary. Often, several approaches used simultaneously are required to maintain euthermia in a bleeding patient. ECMO, extracorporeal membrane oxygenation; $\mathrm{CV}$, cardiovascular; TEMP, temperature.

Although forced warm air systems function well, several other devices to maintain or increase core temperature are available. Circulating water systems may be more efficient than forced-air devices, perhaps because the former provide heat to both anterior and posterior body surfaces. A special garment that circulates warm water through flexible devices that can be wrapped around the torso and extremities may transfer heat more efficiently than forced air devices. Convective air blankets and under-body warming systems may also be beneficial. 
Heating intravenous fluids is critically important in the prevention and treatment of hypothermia [20]. As noted, infusion of room-temperature or colder crystalloid or blood products can reduce core temperature rapidly. It is worthwhile to warm all infused fluids when treating hemorrhage. To this end, when massive infusions of blood or crystalloid are necessary, rapid warmer-infusers are available that can deliver warmed fluids at more than $1 \mathrm{~L} / \mathrm{min}$. Staff in the operating room and delivery room should be familiar with how to use all of the hospital's fluid warming products. Generally, fluids should be infused at $37^{\circ} \mathrm{C}$ $42^{\circ} \mathrm{C}$. (Excessive heating of blood or use of a microwave oven can cause hemolysis). In the operating room, irrigation of the stomach with warm water or saline administered via nasogastric tube works well, as does irrigation of the bladder. Pouring warm fluid into the open abdominal cavity is quite effective in transferring heat to the patient.

In very profound or obdurate cases, cardiopulmonary bypass, extracorporeal membrane oxygenation (ECMO) or a veno-venous bypass system can be used, although these are rarely necessary for hemorrhagic hypothermia that develops in hospital. The optimal speed with which rewarming should be accomplished is unclear, and may vary according to circumstances.

Although they are uncommon, the rewarming process may impose some risks. These include hyperkalemia, a mismatch between tissue oxygen requirements and delivery, and systolic dysfunction. In general, gradual rewarming is preferable unless there is uncontrollable coagulopathy. Thermal burns can occur if equipment malfunctions or is not used properly. Core warming can be delayed when circulation is inadequate, emphasizing the importance of promptly restoring hemodynamic function when treating severe hemorrhage.

\section{Therapeutic Hypothermia}

Concerns about hypothermia in the bleeding patient may seem counterintuitive, because intentionally imposed hypothermia has been used to advantage during cardiac and neurologic surgery, and in the treatment of several acute medical problems [21]. Moreover, victims of cold water near-drowning are sometimes resuscitated after extraordinary immersion times; presumably these survivors were served well by the decreased metabolic rate imposed by a low core temperature. Carefully induced therapeutic hypothermia has even been suggested as a potential treatment for patients in shock. All of the latter situations are, however, physiologically distinct from the accidental loss of heat that can occur during acute massive blood loss, and the role of induced hypothermia in the management of acute hemorrhagic shock remains to be determined.

\section{Coda}

Maternal death from hemorrhagic shock remains among the most common causes of pregnancy-related mortality, even when bleeding occurs in the milieu of a modern hospital. Our approaches to the management of severe blood loss 
and its sequelae have improved considerably; but hypothermia, an often overlooked complication of massive hemorrhage and vigorous fluid resuscitation can be a major contributor to maternal mortality. Hypothermia is a sinister and silent killer, and any physician managing severe obstetric bleeding should be aware of it and take necessary measures to prevent it or to treat it promptly if it occurs.

Monitoring core temperature in the operating room is usually in the purview of the anesthesiologist. It is, nevertheless, a shared responsibility, and the surgeon and anesthesiologist must communicate with each other about changes in temperature and decide on the best therapeutic option. Often this will involve using several methods of warming simultaneously.

For the obstetrician managing hemorrhage initially it is vitally important that serious attempts to prevent or treat heat loss be made, based on frequent core temperature monitoring. Such efforts may obviate the need for operative intervention. If urgent surgery is necessary, initiating anesthesia in a euthermic patient rather than one with disrupted thermoregulation reduces its risks considerably. Once a hypothermic patient has been rewarmed, she still requires careful surveillance until complete metabolic homeostasis has been restored. Observation for late complications such as infection or cardiac compromise is necessary.

\section{Contributions to Authorship}

WRC is the sole author. He conceptualized, planned and wrote the manuscript.

\section{Ethics Approval}

As a review article that did not involve human or animal subjects or medical records, this project was exempt from ethics committee (IRB) approval.

\section{Funding}

No funding was applied to the production of this review.

\section{Conflicts of Interest}

The author reports no conflict of interest.

\section{References}

[1] Sessler, D.I. (2008) Temperature Monitoring and Perioperative Thermoregulation. Anesthesiology, 109, 318-338. https://doi.org/10.1097/ALN.0b013e31817f6d76

[2] Tsuji, T. (1987) Patient Monitoring during and after Open Heart Surgery: An Improved Deep Body Thermometer. Medical Progress through Technology, 12, 25-38. https://doi.org/10.1007/978-94-009-3361-3_4

[3] Rajagopalan, S., Mascha, E., Na, J. and Sessler, D.I. (2008) The Effects of Mild Perioperative Hypothermia on Blood Loss and Transfusion Requirement. Anesthesiology, 108, 71-77. https://doi.org/10.1097/01.anes.0000296719.73450.52 
[4] Sessler, D.I. (1997) Mild Perioperative Hypothermia. New England Journal of Medicine, 336, 1730-1737. https://doi.org/10.1056/NEJM199706123362407

[5] Trentzsch, H., Nienaber, U., Behnke, M., Lefering, R. and Piltz, S. (2014) Female Sex Protects from Organ Failure and Sepsis after Major Trauma Hemorrhage. Injury, 45, S20-S28.

[6] Charkoudian, N. and Stachenfeld, N. (2016) Sex Hormone Effects on Autonomic Mechanisms of Thermoregulation in Humans. Autonomic Neuroscience, 196, 75-80. https://doi.org/10.1016/j.autneu.2015.11.004

[7] Morison, S.F., Nakamura, K. and Madden, C.J. (2008) Central Control of Thermogenesis in Mammals. Experimental Physiology, 93, 773-797.

https://doi.org/10.1113/expphysiol.2007.041848

[8] Dietrichs, E.S., Sager, G. and Tvieta, T. (2016) Altered Pharmacological Effects of Adrenergic Agonists during Acute Hypothermia. Scandinavian Journal of Trauma, Resuscitation and Emergency Medicine, 24, 143-151.

https://doi.org/10.1186/s13049-016-0339-8

[9] Gubler, K.D., Gentiello, L.M., Hassantash, S.A., and Maier, R.V. (1994) The Impact of Hypothermia on Dilutional Coagulopathy. Journal of Trauma, 36, 847-851. https://doi.org/10.1097/00005373-199406000-00015

[10] Brohi, K., Cohen, M.J., Ganter, M.T., Matthay, M.A., Mackersie, R.C. and Pittet, J.F. (2007) Acute Traumatic Coagulopathy: Initiated through Hypoperfusion; Modulated through Protein C Pathway? Annals of Surgery, 245, 812-818.

[11] Watts, D.D., Trask, A., Soeken, K., Perdue, P., Dols, S. and Kaufmann, C. (1998) Hypothermic Coagulopathy in Trauma: Effect of Varying Levels of Hypothermia on Enzyme Speed, Platelet Function, and Fibrinolytic Activity. Journal of Trauma, 44, 846-854. https://doi.org/10.1097/00005373-199805000-00017

[12] Martini, W.Z. (2009) Coagulopathy by Hypothermia and Acidosis: Mechanisms of Thrombin Generation and Fibrinogen Availability. Journal of Trauma, 67, 202-208. https://doi.org/10.1097/TA.0b013e3181a602a7

[13] Frank, S.M., Fleisher, L.A., Breslow, M.J., Higgins, M.S., Olson, K.F., Kelly, S., et al. (1997) Perioperative Maintenance of Normothermia Reduces the Incidence of Morbid Cardiac Events: A Randomized Clinical Trial. JAMA, 277, 1127-1134. https://doi.org/10.1001/jama.1997.03540380041029

[14] Lenhardt, R., Marker, E., Goll, V., Tschernich, H., Kurz, A., Sessler, D.I., et al. (1997) Mild Intraoperative Hypothermia Prolongs Postanesthetic Recovery. Anesthesiology, 87, 1318-1323. https://doi.org/10.1097/00000542-199712000-00009

[15] Hildebrand, F., van Griensven, M., Giannoudis, P., Schreiber, T., Frink, M., Probst, C., et al. (2005) Impact of Hypothermia on the Immunologic Response after Trauma and Elective Surgery. Surgical Technology International, 14, 41-50.

[16] Lenhardt, R. (2010) The Effect of Anesthesia on Body Temperature Control. Frontiers in Bioscience, 2, 1145-1154. https://doi.org/10.2741/s123

[17] Madrid, E., Urrútia, G., Roqué, I., Figuls, M., Pardo-Hernandez, H., Campos, J.M., et al. (2016) Active Body Surface Warming Systems for Preventing Complications Caused by Inadvertent Perioperative Hypothermia in Adults. The Cochrane Database of Systematic Reviews, 4, Article ID: CD009016. https://doi.org/10.1002/14651858.CD009016.pub2

[18] Sultan, P., Habib, A.S., Cho, Y. and Carvalho, B (2015) The Effect of Patient Warming during Caesarean Delivery on Maternal and Neonatal Outcomes: A Meta-Analysis. British Journal of Anaesthesia, 115, 500-510.

https://doi.org/10.1093/bja/aev325 
[19] Bräuer, A. and Quintel, M. (2009) Forced-Air Warming: Technology, Physical Background and Practical Aspects. Current Opinion in Anesthesiology, 22, 769-774. https://doi.org/10.1097/ACO.0b013e328331d134

[20] Campbell, G., Alderson, P., Smith, A.F. and Warttig, S. (2015) Warming of Intravenous and Irrigation Fluids for Preventing Inadvertent Perioperative Hypothermia. The Cochrane Database of Systematic Reviews, 4, Article ID: CD009891.

[21] Lampe, J.W. and Becker, L.B. (2011) State of the Art in Therapeutic Hypothermia. Annual Review of Medicine, 62, 79-93.

https://doi.org/10.1146/annurev-med-052009-150512 